\title{
Las exportaciones de hidrocarburos y el crecimiento económico en Colombia 1960-2016: un análisis según la hipótesis de la enfermedad holandesa
}

\author{
Johnny Humberto Vargas Restrepo* \\ Juan Pablo Saldarriaga Muñoz ${ }^{* *}$
}

Fecha de recepción: 4 de diciembre de 2019

Fecha de aceptación: 3 de mayo de 2020

Resumen: La hipótesis de la "enfermedad holandesa" prevé que existe una relación negativa entre las exportaciones de bienes primarios y el sector transable de la economía (principalmente la industria), de ese modo se termina afectando el crecimiento económico. En este trabajo se analiza este fenómeno para el caso de las principales exportaciones Colombia, petróleo y carbón. Por medio de series de tiempo se modeló el efecto de las exportaciones de estos combustibles sobre el tipo de cambio, el sector transable de economía y la tasa de crecimiento económico colombiano durante el periodo 1960-2016, y los resultados muestran que se cumple esa hipótesis. Adicionalmente se testeó si los procesos de apertura causaron también efectos similares a los de la enfermedad holandesa, pero no se encontró evidencia de ello.

Palabras clave: crecimiento económico; combustibles; enfermedad holandesa; recursos naturales; industria; tipo de cambio; exportaciones.

Clasificación JEL: O13, O40, Q33, Q35, Q40.

Cómo citar este artículo/ To reference this article / Comment citer cet article / Para citar este artigo:

Vargas-Restrepo, J., \& Saldarriaga-Muñoz, J. (2020). Las exportaciones de hidrocarburos y el crecimiento económico en Colombia 1960-2016: un análisis según la hipótesis de la enfermedad holandesa. Apuntes Del Cenes, 39(70). Págs. 167 - 182. https://doi.org/10.19053/01203053.v39.n70.2020.10421

\footnotetext{
* Magíster en Ciencias Económicas, profesor asistente de la Facultad de Ciencias Económicas y Administrativas de la Universidad del Tolima. Colombia. Correo electrónico: jhvargasre@ut.edu.co (i) https://orcid.org/0000-00026999-941X Contacto de correspondencia.

** Magíster en Economía, profesor asistente de la Facultad de Ciencias Económicas y Administrativas de la Universidad del Tolima. Colombia. Correo electrónico: jpsaldarriagam@ut.edu.co (i) https://orcid.org/0000-0002-2445-0036.
} 


\title{
Hydrocarbon Exports and Economic Growth in Colombia 1960- 2016: An Analysis according to the Dutch Disease Hypothesis
}

\begin{abstract}
The "Dutch disease" hypothesis predicts that there is a negative relationship between exports of primary goods and the tradable sector of the economy (mainly industry), thus ending up affecting economic growth. This work analyzes this phenomenon in the case of the main Colombian exports, oil and coal. The effect of exports of these fuels on the exchange rate, the tradable sector of the economy and the rate of Colombian economic growth during the period 1960-2016 was modeled using time series, and the results show that this hypothesis is fulfilled. Additionally, it was tested whether the opening processes also caused effects similar to those of the Dutch disease, but no evidence of this was found.
\end{abstract}

Keywords: economic growth, fuels, Dutch disease, natural resources, industry, exchange rate, exports. 


\section{INTRODUCCIÓN}

En las últimas décadas, el petróleo y el carbón han sido el principal renglón de las exportaciones del país, así como el sector de mayor registro en inversión extranjera directa. El auge de estas exportaciones propició un aumento en el grado de apertura de la economía y una diversificación de la cesta exportadora del país, antes con mayor dependencia del café. Adicionalmente incrementaron los ingresos fiscales, de modo que no fueron un fenómeno transitorio (Vargas, 2010).

Ha existido un debate recurrente en torno a la relación de la abundancia de recursos naturales y su efecto sobre el desempeño de la economía, especialmente el efecto del sector minero energético sobre el crecimiento económico. Este trabajo aborda esa discusión mediante la hipótesis de la "enfermedad holandesa" y evalúa a través de análisis de series de tiempo el efecto de las exportaciones de estos combustibles fósiles sobre el tipo cambio, después sobre el sector transable y finalmente sobre la tasa de crecimiento económico de Colombia durante el periodo 1960-
2016, y si dichos efectos se ajustan a dicha hipótesis.

Diferentes autores aducen que los procesos de apertura también afectaron el desempeño de la economía, por ello en este trabajo se involucra ese análisis. Específicamente se estiman los efectos que tuvieron los procesos de apertura comercial y financiera sobre el sector externo y si estos también causaron los síntomas de enfermedad holandesa, tales como la apreciación del tipo de cambio y la disminución de la participación del sector transable en el PIB.

El presente trabajo se desarrolla de la siguiente manera: primero se presentan algunas apreciaciones generales de la enfermedad holandesa. A continuación, se examinan antecedentes de este fenómeno en Colombia, incluyendo además reseñas de los desajustes en el sector transable asociados a los procesos de apertura. Luego se procede a estimar por medio de series de tiempo dicha hipótesis para el caso de la economía colombiana, posteriormente se lleva a cabo el análisis de resultados y finalmente se presentan las conclusiones. 


\section{APRECIACIONES SOBRE LA EN- FERMEDAD HOLANDESA}

Un hallazgo importante de minerales o hidrocarburos, un avance en productividad o precios altos en el mercado internacional, se denomina bonanza exportadora o auge del sector. Esta situación se suele asociar a la ocurrencia de un "boom" exportador de los bienes primarios, cuyo incremento en el flujo de divisas genera una apreciación real y esta a su vez afecta la competitividad de los demás sectores transables frente a sus competidores en el exterior. Como consecuencia se reduce la producción de transables (principalmente la industria y la agricultura) en el ámbito nacional y parte de sus factores de producción se desplazan al sector de bienes no transables que dependen principalmente de las condiciones del mercado local (Corden \& Neary, 1982; Posada, 1994; De Tray, 1994; Gelb, 1988; Rodríguez \& Sachs, 1999).

Según Sachs y Andrew (1997), si un país se especializa en la producción de bienes primarios y bienes no transables, termina afectando su dinámica de crecimiento, pues estos sectores emplean poco capital humano y tecnología en su producción. También habría que agregar que el sector minero energético y algunos renglones del sector no transable no son grandes empleadores de mano de obra, como sí es el caso de la industria.
En este sentido, el trabajo de Perla (2005) analiza diferentes estudios sobre la relación entre abundancia de recursos naturales, específicamente petróleo y minerales, con el crecimiento económico. En uno de sus enfoques, denominado de precios relativos (otra manera para denominar los síntomas de la enfermedad holandesa), reseña como la especialización en estos bienes primarios afecta la tasa de crecimiento.

Perla (2005) también reseña estudios que cuestionan algunos postulados de la enfermedad holandesa, por ejemplo, los referentes a los movimientos de los factores de producción durante una bonanza, y, por tanto, el sector industrial podría crecer a la par con el sector minero energético. Finalmente concluye que este enfoque no es suficiente para explicar por qué los recursos minero-energéticos afectan el crecimiento de algunos países, pero a otros no (pp. 16-20). Se requieren otros enfoques para explicar la diferencia de los efectos de este sector en cada país.

En esa última perspectiva, Perry y Olivera (2012) reseñan que existen casos individuales de maldición de recursos naturales, especialmente de países ricos en petróleo y minerales, pero los mecanismos para explicar este efecto podrían ser de naturaleza política o económica. Se sugiere que la literatura ha enfatizado en el papel que han tenido las instituciones sobre el desempeño económico (p. 85). Se reseñan casos de países que tuvieron síntomas de enfer- 
medad holandesa en algunas épocas de bonanza, pero luego los superaron en las bonanzas recientes (pp. 16 y 17).

Haussman y Rigobon (2003) afirman que no está claro, desde el punto de vista empírico, que las industrias basadas en recursos naturales sean menos intensivas en mano de obra calificada y conocimiento que otras industrias. Según Perry y Olivera (2012), los defensores de la hipótesis de la enfermedad holandesa pocas veces intentan ofrecer evidencia de la "superioridad" del sector transable diferente al de recursos naturales sobre el sector no transable y de recursos naturales (p. 86).

Es relevante entonces analizar para el caso colombiano cuál ha sido el efecto del sector minero-energético sobre los demás sectores de la economía. Por tanto, se procede a revisar antecedentes y luego se hacen estimaciones que respondan esta inquietud.

\section{ANTECEDENTES EN COLOMBIA}

En la década de los ochenta con el hallazgo de Caño Limón y con la explotación de la mina del Cerrejón ocurre un profundo cambio en la estructura del sector externo, en términos de crecientes exportaciones e inversión extranjera en los subsectores de petróleo y carbón. $\mathrm{Y}$ desde entonces su contribución a la economía colombiana ha sido relevante, a tal grado que en la segunda mitad de los noventa el país duplicó el volumen de sus exportaciones de petróleo, desplazando al segundo lugar al café, mismas que caerían al tercer lugar antes del fin de la década, esta vez desplazadas por las crecientes exportaciones de carbón ${ }^{1}$. Dada la importancia de este sector en la economía colombiana, es conveniente analizar sus efectos sobre los demás sectores de la economía.

Vargas (2010) analiza el fenómeno de la enfermedad holandesa para Colombia durante el periodo 1970-2007. Estima la relación entre exportaciones de hidrocarburos con tipo de cambio real, luego con PIB transable y finalmente con PIB industrial, para ver si estas exportaciones afectaban estas variables, pero no encontró una relación significativa entre las variables. Del mismo modo, estimó la relación entre dichas exportaciones con el crecimiento económico en este periodo, pero tampoco encontró una relación negativa como predice la hipótesis de enfermedad holandesa (p. 130).

Clavijo et al. (2012) estudian el fenómeno de la desindustrialización en Colombia desde dos enfoques: el primero, llamado las tendencias seculares, que se manifiestan en el crecimiento de la manufactura como porcentaje en el PIB hasta un límite y luego empieza caer mientras incrementa la participación del sector servicios. Todo ello como consecuencia del acelerado crecimiento de la productividad e innovaciones en

1 Observaciones como esta pueden verse en Greco-Banco de la República (2002) y Vargas (2010). 
otros sectores. Este cambio es gradual y se va dando una expansión del sector servicios. El avance tecnológico impulsa el sector terciario con énfasis en servicios financieros.

El segundo enfoque se refiere a la denominada enfermedad holandesa, es decir, que el auge en el sector minero energético apreció la tasa de cambio real y de esta manera se afectó la producción de transables, en especial la manufactura.

Este trabajo reseña el fenómeno de la desindustrialización en Latinoamérica, donde a finales de los setenta se estanca la producción industrial para continuar decayendo. Pero no se debe principalmente por la tendencia secular, sino que se debe al agotamiento de la primera fase de sustitución de importaciones (manufactura liviana) y las dificultades estructurales para competir externamente.

Particularmente en Colombia se muestra que el país no había alcanzado niveles de productividad y de desarrollo (medido por PIB per cápita) como los países denominados desarrollados y por ello la desindustrialización se explica más por el fenómeno de la enfermedad holandesa. En sus resultados muestra un efecto negativo de la relación de exportaciones minero-energéticas sobre la relación valor agregado industrial. La variable PIB de servicios también resultó significativa, dando cierto sustento a la hipótesis secular, pero es un efecto marginal. El análisis se realizó para el periodo 1965-2010.
Ojeda et al. (2013) reseñan estudios sobre enfermedad holandesa y hacen hincapié en los efectos de desindustrialización y la mejora en el sector no transable. Sin embargo, se muestra que un auge del sector exportador puede disminuir el costo del endeudamiento y además que las remesas recibidas por el país mejoran el bienestar de las familias. Además, se mencionan estudios donde se muestra que el efecto de un auge del sector de recursos naturales podría incentivar a la industria, si el país se especializa en industrias que utilizan intensamente el recurso natural como insumo (pp. 565-567).

En este trabajo hay estimaciones entre 1996 y 2011, que muestran como un aumento en la productividad del sector minero energético disminuye temporalmente la producción de manufacturas, aunque eleva el bienestar por el mayor consumo de los hogares.

Según Kalmanovitz (2015), las bonanzas cafeteras en los años setenta y ochenta, las bonanzas del sector minero energético desde los años ochenta y algunos periodos de auge exportador de drogas ilegales, contribuyeron a la revaluación, síntoma de la enfermedad holandesa (p. 190). Aunque también se reseña el papel de la afluencia de capitales sobre la revaluación.

García (2005) encontró que hay una relación positiva en la tasa de producción de petróleo y la tasa de crecimiento del PIB. Por otro lado, diferentes autores 
reseñan que no había evidencia de enfermedad holandesa (Garay, 2013; Piraján et al., 2012; Vargas, 2010), por lo que se deben seguir examinando otras causas del comportamiento negativo del sector transable.

Por tanto, el auge exportador por sí solo no necesariamente explica el efecto negativo sobre los demás sectores transables, pues este tiene varios matices, es un fenómeno multicausal. Diferentes trabajos han señalado que los efectos de la apertura comercial y financiera generaron efectos adversos sobre el sector transable.

\section{APERTURA ECONÓMICA Y EFECTOS SOBRE EL SECTOR TRANSABLE}

Desde los años ochenta se dieron simultáneamente auges del sector minero-energético y procesos de apertura. La apertura comercial unilateral de los noventa condujo a un crecimiento mayor de las importaciones sobre las exportaciones (Ocampo, 2015, p. 312; Vargas, 2010, p. 73).

Para Ocampo (2015), la mayor competencia de las importaciones y la caída de los precios relativos de los productos industriales en el mercado interno promovieron el proceso de desindustrialización (p. 319). El sector agropecuario también tuvo una disminución en su participación desde los años ochenta, debido a los efectos de la apertura, la revaluación y los bajos precios internacionales (p. 324).

Por otro lado, la apertura financiera permitió una importante entrada de capitales que condujeron a una apreciación real en la década de los noventa (Ffrench-Davis, 1997; Montenegro et al., 2001). Esta afluencia de capitales, que incluye incremento del endeudamiento, también genera la apreciación y por tanto se ocasionan desajustes entre los sectores transables y no transables, como predice la hipótesis de la enfermedad holandesa (Urrutia, 1994).

Ffrench-Davis (1997) reseña algunas características de los capitales que ingresaron: estos se dirigieron al consumo, al sector no transable y fue poco lo que llegó al sector transable (diferente al sector minero energético), que debía ser el sector que haría sostenible el endeudamiento. Ocampo (2015) muestra que en el periodo 1994-2003 la IED se concentró en el sector servicios y después de 2004 más del $50 \%$ de esta se dirigió al sector minero energético (p. 307).

Según Kalmanovitz (2015), la liberación financiera en los años noventa incentivó la revaluación e hizo más volátil la tasa de cambio real y de esa manera más vulnerable la economía a crisis financieras internacionales como las ocurridas a finales de los años noventa (p. 192). Ocampo (2015) considera que esta liberación cambiaria y financiera 
sometió a la economía colombiana a ciclos más agudos y por tanto generó mayor inestabilidad (p. 326).

Goda y Torres (2015) examinaron el efecto de los flujos de capitales en Colombia a inicios de este siglo. El objetivo fue analizar de qué manera estos flujos incidieron sobre el tipo de cambio y luego los efectos de la tasa cambio real sobre los cambios en la estructura de los sectores transables y no transables. Especialmente se examina el efecto de los flujos de capital que llegan al sector minero energético y si estos pudieron ocasionar la enfermedad holandesa.

Los autores reseñan diversos estudios que no han mostrado una evidencia única a favor de que mayores flujos de capital causen mayor apreciación cambiaria. Pero el análisis realizado en este trabajo para Colombia entre 2003 y 2012 muestra señales de enfermedad holandesa, debido a la disminución de la participación del sector transable mientras ocurría el auge del sector minero energético. Los flujos de capital apreciaron la TCR, tanto los flujos de IED del sector minero energético como los que llegan a otros sectores. El efecto de la entrada de capitales es mayor desde el segundo semestre del 2002, cuando inicia el boom de precios internacionales.

El estudio reseñado concluye que probablemente la IED en el sector minero energético no tuvo efectos importantes sobre la productividad factorial y que este sector tiene una limitada relación con lo demás sectores de la economía.

Hasta aquí se han revisado trabajos que muestran los efectos del sector minero energético y de los procesos de apertura sobre el sector transable en especial en el periodo comprendido desde finales de los años ochenta en adelante. El presente trabajo muestra un horizonte más amplio (1960-2016) para explicar los efectos a largo plazo, como se requiere en análisis de crecimiento económico. Adicionalmente se intenta analizar efectos de variables diferentes a las exportaciones de combustibles que también podrían producir los síntomas de enfermedad holandesa, tales como variables relacionadas con la apertura comercial y financiera.

\section{ESTIMACIONES DE LA HIPÓ- TESIS DE LA ENFERMEDAD HOLANDESA EN EL PERIODO 1960-2016}

Como se indicó anteriormente, es preciso mostrar los efectos de las exportaciones de combustibles sobre el tipo de cambio, el sector transable y el crecimiento para evaluar los síntomas de la enfermedad holandesa. Se procede entonces a describir las relaciones por estimar en este enfoque y luego se muestran los resultados obtenidos.

En la ecuación 1 se establece una relación de las exportaciones de hidrocarburos con el tipo de cambio, con 
el objetivo de examinar si se da una apreciación cambiaria, síntoma de la enfermedad holandesa. Adicionalmente se pretende testear el efecto de indicadores de apertura comercial y financiera, para ver si afectan el tipo de cambio. $E R$ corresponde a la variable de tipo de cambio, EXPFUELL a las exportaciones de petróleo y carbón, OPEN índice de apertura comercial y la variable $A F$ representa la apertura financiera. Como se revisó antes, los flujos de capitales también pueden causar apreciación cambiaria, por tanto, se intentó incluir variables como indicadores de entrada de capitales, IED, flujos de deuda, etc. Sin embargo, sus efectos no fueron significativos, por ello se excluyen luego de los resultados de las estimaciones.

$$
E R_{t}=\alpha_{0}+\alpha_{1} E X P F U E L_{t}+\alpha_{2} O P E N_{t}+\propto_{3} A F_{t}+\varepsilon_{t}
$$

La ecuación 1 se estima en tasas de variación porcentual de cada una de las variables, como se muestra en la Tabla 1. En la ecuación 2, GDPR corresponde a la tasa de crecimiento del PIB transable y este se calcula como el cociente de la suma del PIB agrícola y de manufacturas respecto al PIB total. Se relaciona con la variación de las exportaciones de petróleo y carbón (VEXPFUEL) para de esta manera testear si estas tuvieron un efecto negativo sobre estos sectores de la producción. También se relaciona la tasa del PIB transable con la variación de la apertura comercial (VOPEN) y financiera $(V A F)$. En este caso se testeó con la variable $O P E N$ y no $A F$ en las estimaciones, pues de nuevo se trató de involucrar otras variables relacionas con la apertura financiera, IED y flujos de deuda externa, pero tampoco resultaron significativas.

$$
\mathrm{GDPTR}_{t}=\alpha_{0}+\alpha_{1} V E X P F U E L_{t}+\alpha_{2} V_{O P E N_{t}}+\alpha_{3} V A F_{t}+\varepsilon_{t}
$$

Si $\alpha 1$ es negativo en ambas ecuaciones, significaría que las exportaciones de hidrocarburos disminuyen el tipo de cambio real y la producción transable, lo cual es síntoma de enfermedad holandesa. Si $\alpha_{2}$ es negativo, significa que la apertura económica también afectó el sector transable.
Para establecer los efectos de las exportaciones de combustibles sobre el crecimiento económico y demás variables que se consideran en la hipótesis de la enfermedad holandesa, se hace la siguiente relación:

$$
\text { GROWTH }_{t}=\alpha_{0}+\alpha_{1} k_{t}+\alpha_{2} h_{t}+\alpha_{3} \text { VEXPFUEL } L_{t}+\alpha_{4} \text { VOPEN }_{t}+\alpha_{5} V E R_{t}+\varepsilon_{t}
$$


Donde GROWTH es crecimiento económico, $\mathrm{k}$ es el crecimiento del capital físico, $h$ es crecimiento del capital humano, VER es la variación porcentual del tipo de cambio, es la variación porcentual de las exportaciones de hidrocarburos y VOPEN es la variación porcentual del indicador de apertura.

El capital físico (k) y el capital humano (h) se toman como las variables de control y se espera un efecto positivo sobre el crecimiento. Si $\alpha 3$ tienen un efecto negativo, entonces se comprobaría un efecto maldición de recursos. El signo de $\alpha 4$ muestra el efecto de la apertura económica sobre el crecimiento y $\alpha 5$ recoge el efecto del tipo de cambio.

Los datos sobre crecimiento económico (GROWTH), exportaciones de combustibles $(E X P F U E L L)$ y bienes transables (GDPTR), fueron tomados de los indicadores de desarrollo humano del Banco Mundial para el período 1960-2016. Los índices de capital físico (k) y humano (h) fueron tomados de Posada y Rojas (2008) para el período 1960 a 2000 y actualizados para el período 2001-2016 según la metodología utilizada por los autores ${ }^{2}$, utilizando para el caso del capital humano la información de los estudiantes en secundaria para Colombia de la UNESCO y la población total y la tasa de mortalidad de adultos del DANE. Mediante el análisis de series de tiempo se realizaron las estimaciones para el periodo 1960-2016.

Para el caso de los datos de tipo de cambio (ER), estos fueron tomados de los indicadores de tipo de cambio nominal del Banco Mundial. Otra alternativa era tomar los datos del índice de tipo de cambio de real del Banco de la República de Colombia, pero solo se disponían datos desde 1975, no para el periodo completo de este análisis. Ahora, para apertura comercial (OPEN) la referencia fue la participación de las exportaciones e importaciones en el PIB y de esta manera los datos también se obtuvieron de los indicadores del Banco Mundial. Mediante el análisis de series de tiempo se realizaron las estimaciones para el periodo 1960-2016.

\section{RESULTADOS}

Para las estimaciones se utilizó Eviews 9.0. Un análisis inicial de las variables arrojó que ER, EXPFUELL, OPEN, K son integradas de orden uno, I(1), GDP$T R, G R O W T H, \mathrm{k}$ y h son integradas de orden cero, I (0). La Tabla 1 muestra los resultados de las estimaciones de

2 El índice de capital físico se construye a partir de la siguiente ecuación: $K_{t}=I_{k, t}+(1-d) K_{t-1}$, donde $I_{k}$ es la inversión en capital físico y $d$ es la tasa de depreciación. Para mayor detalle véanse Posada y Rojas (2008, p. 20) El índice de capital humano se construye a partir de la siguiente ecuación de movimiento:

$H_{t}=I_{h, t}+(1-m) H_{t-1}$, donde $I_{h}$ es la inversión en capital humano y $m$ es la tasa de mortalidad; como proxy de inversión se utiliza la relación entre alumnos matriculados en secundaria y la población total. Para mayor detalle véanse Posada y Rojas (2008, p. 15). 
las ecuaciones [1] y $[2]^{3}$. La ecuación [1] es útil para ver si las exportaciones de combustibles generaban apreciación cambiaria y de ese modo afectaban la producción de transables, pero no se encontró una relación significativa. La apreciación de la moneda que afecta al sector transable es un mecanismo de transmisión muy relevante de la enfermedad holandesa, pero en este caso no se pudo establecer ese canal de transmisión.

En la estimación de la ecuación [2] se evidencia que el incremento de las ex- portaciones de combustibles afectaba al sector transable, lo que indica síntomas de enfermedad holandesa. Como se mencionó en otros trabajos, la apertura económica afectó el sector transable, pero en este caso, el efecto del índice de apertura sobre el sector transable es positivo, como se indica en la Tabla $1^{4}$.

También se había indicado que la afluencia de capitales generaba apreciación y afectaba al sector transable, pero en este caso las variables asociadas a ello no resultaron significativas para evaluar ese canal de transmisión.

Tabla 1. Estimaciones relaciones 1 y 2

\begin{tabular}{|c|c|c|c|c|c|}
\hline $\begin{array}{c}\text { Variable } \\
\text { Dependiente: }\end{array}$ & DLOG(ER) & DLOG(ER) & $\begin{array}{c}\text { Variable } \\
\text { Dependiente: }\end{array}$ & D(GDPTR) & $\mathrm{D}$ (GDPTR) \\
\hline & ER (1) & ER (2) & & TR (1) & TR (2) \\
\hline DLOG(ER(-1)) & $\begin{array}{c}0.5596^{* * *} \\
(0.1105)\end{array}$ & $\begin{array}{c}0.5916^{* * *} \\
(0.1182)\end{array}$ & $\mathrm{D}(O P E N)$ & $\begin{array}{c}0.2348 \text { ** } \\
(0.0885)\end{array}$ & $\begin{array}{l}0.1519 \text { ** } \\
(0.0740)\end{array}$ \\
\hline DLOG(OPEN) & $\begin{array}{l}0.2994^{*} \\
(0.1660)\end{array}$ & & DEUDA_EXP & $\begin{array}{c}0.0029 \\
(0.0035)\end{array}$ & \\
\hline $\begin{array}{l}\text { DLOG(EXP_ } \\
\text { FUEL) }\end{array}$ & & $\begin{array}{c}0.0248 \\
(0.0376) \\
\end{array}$ & $\mathrm{D}$ (EXP_FUEL) & & $\begin{array}{c}-0.0606^{* *} \\
(0.0291) \\
\end{array}$ \\
\hline C & $\begin{array}{c}0.0486 \text { ** } \\
(0.0173)\end{array}$ & $\begin{array}{c}0.0487^{* *} \\
(0.0181)\end{array}$ & C & $\begin{array}{l}-1.1838 \text { * } \\
(0.6778)\end{array}$ & $\begin{array}{c}-0.6045^{* * *} \\
(0.1624)\end{array}$ \\
\hline R-squared & 0.3561 & 0.3381 & R-squared & 0.1552 & 0.1677 \\
\hline F-statistic & 14.3774 & 12.7685 & F-statistic & 3.9488 & 4.7355 \\
\hline $\begin{array}{l}\text { Prob(F- } \\
\text { statistic) }\end{array}$ & 0.0000 & 0.0000 & $\begin{array}{l}\text { Prob(F- } \\
\text { statistic) }\end{array}$ & 0.0266 & 0.0134 \\
\hline Akaike & -1.8927 & -1.8342 & Akaike & 3.2303 & 3.1268 \\
\hline Schwarz & -1.7832 & -1.7226 & Schwarz & 3.3496 & 3.2416 \\
\hline Hannan-Quinn & -1.8504 & -1.7913 & Hannan-Quinn & 3.2750 & 3.1705 \\
\hline Durbin-Watson & 2.1379 & 2.0074 & Durbin-Watson & 1.9215 & 1.7969 \\
\hline
\end{tabular}

3 Dado que las variables de la ecuación [1] son integradas de orden uno, se procedió a estimar el modelo en primeras diferencias del logaritmo de las variables, lo que representaría en el caso de la variable dependiente la depreciación de la moneda. Además, dado que se encontró evidencia de autocorrelación de primer orden, se introdujo un rezago de la variable dependiente, lo que puede estar asociado a un comportamiento inercial de la misma.

4 En los modelos presentados en la Tabla 1 no se encontró evidencia de presencia de heterocedasticidad o autocorrelación, para lo cual se realizaron tanto test de White, Breucsh-Pagan-Godfrey, ARCH y Breusch-Godfrey. Tampoco se encontró evidencia de cambio estructural, para lo cual se llevó a cabo el test de Quandt-Andrews. 
Para la ecuación 3 se hicieron diferentes estimaciones del modelo de corrección de errores con rezagos distribuidos usando el paquete econométrico, los resultados a corto y largo plazo se muestran en la Tabla 2 y en la Tabla 3 respectivamente.

Tabla 2. Estimación a corto plazo de la relación 3

\begin{tabular}{|c|c|c|c|c|}
\hline & Mod (1) & Mod (2) & Mod (3) & $\operatorname{Mod}(4)$ \\
\hline $\mathrm{AR}(1)$ & $\begin{array}{c}0.3225^{* *} \\
(0.1331)\end{array}$ & $\begin{array}{c}0.3462 \text { ** } \\
(0.1287)\end{array}$ & $\begin{array}{l}0.3515^{* *} \\
(0.1360)\end{array}$ & $\begin{array}{c}0.3071 \text { ** } \\
(0.1394)\end{array}$ \\
\hline DLOG(K) & $\begin{array}{c}56.7398 * * * \\
(12.7180)\end{array}$ & $\begin{array}{c}60.4823 * * * \\
(13.6022)\end{array}$ & $\begin{array}{c}54.4357^{* * *} \\
(13.1158)\end{array}$ & $\begin{array}{c}61.5071 \text { *** } \\
(13.0435)\end{array}$ \\
\hline $\operatorname{DLOG}(\mathrm{H})$ & $\begin{array}{c}80.9673^{* * *} \\
(23.5924)\end{array}$ & $\begin{array}{l}67.7075^{* *} \\
(25.0491) \\
\end{array}$ & $\begin{array}{c}84.8671 \text { *** } \\
(24.5626)\end{array}$ & $\begin{array}{c}69.7903 \text { ** } \\
(23.9833) \\
\end{array}$ \\
\hline DLOG(ER) & $\begin{array}{c}-7.1917^{* *} \\
(2.6838)\end{array}$ & $\begin{array}{c}-4.8888 \text { * } \\
(2.7698)\end{array}$ & $\begin{array}{c}-7.2167^{* *} \\
2,714075\end{array}$ & $\begin{array}{c}-5.7760^{* *} \\
(2.7684)\end{array}$ \\
\hline DLOG(EXP_FUEL) & & $\begin{array}{l}-1.1579 \text { * } \\
(0.6927) \\
\end{array}$ & & \\
\hline DLOG(XC) & & & & $\begin{array}{c}-0.6113 \\
(0.6587) \\
\end{array}$ \\
\hline DLOG(XR) & & & & $\begin{array}{c}3.0845 \\
(1.9906)\end{array}$ \\
\hline DLOG(OPEN) & $\begin{array}{c}7.2466 \text { ** } \\
(3.0373)\end{array}$ & & & \\
\hline DLOG(OPENC) & & & $\begin{array}{l}-0.6457 \\
(0.9387) \\
\end{array}$ & \\
\hline DLOG(OPENR) & & & $\begin{array}{c}5.9923 \text { ** } \\
(2.1793)\end{array}$ & \\
\hline $\mathrm{R} 2$ & 0,2996 & 0,2603 & 0,3433 & 0,2749 \\
\hline $\mathrm{F}$ & 33,8925 & 27,0093 & 26,7651 & 25,9178 \\
\hline P-valor(F) & 0,0000 & 0,0000 & 0,0000 & 0,0000 \\
\hline Akaike & 4,2113 & 4,3014 & 4,2202 & 4,3187 \\
\hline Schwarz & 4,4302 & 4,5244 & 4,4804 & 4,5789 \\
\hline Hannan-Quinn & 4,2959 & 4,3871 & 4,3203 & 4,4188 \\
\hline Durbin-Watson stat & 2,0872 & 2,0849 & 2,1051 & 2,1033 \\
\hline
\end{tabular}

En la Tabla 3, los valores resaltados corresponden a los efectos a largo plazo de cada una de las variables. Los signos de las variables de control asociadas al capital físico y humano son positivos y estadísticamente significativos en todos los casos y es el resultado esperado.

En el caso del tipo de cambio (ER), una depreciación del peso frente al dólar provoca una reducción en el crecimien- to, por ejemplo, según los resultados de la columna Mod (1) de la Tabla 3, por cada $1 \%$ que se devalúe la moneda, el crecimiento se reduce el $0.072 \%$ a corto plazo y $0.106 \%$ a largo plazo. Este resultado es diferente a lo esperado, pues un síntoma de enfermedad holandesa es que la apreciación de tipo de cambio (no la depreciación) reduce el crecimiento. 
A partir de los resultados de la columna Mod (2) de la Tabla 3, por cada $1 \%$ que aumenten las exportaciones de combustibles, el crecimiento se reduce en $0.115 \%$ a corto plazo y 0.18 a largo plazo, lo cual indicaría el efecto negativo que se predice en la hipótesis de maldición de recursos naturales.

Tabla 3. Estimaciones a corto y largo plazo de la relación 3

\begin{tabular}{|c|c|c|c|c|}
\hline & Mod (1) & Mod (2) & Mod (3) & Mod (4) \\
\hline $\mathrm{AR}(1)$ & 0,3225 & 0,3462 & 0,3515 & 0,3071 \\
\hline \multirow{2}{*}{ DLOG(K) } & 56,7398 & 60,4823 & 54,4357 & 61,5071 \\
\hline & 83,7488 & 92,5089 & 83,9409 & 88,7676 \\
\hline \multirow{2}{*}{$\operatorname{DLOG}(\mathrm{H})$} & 80,9673 & 67,7075 & 84,8671 & 69,7903 \\
\hline & 119,5089 & 103,5600 & 130,8668 & 100,7220 \\
\hline \multirow{2}{*}{ DLOG(ER) } & $-7,1917$ & $-4,8888$ & $-7,2167$ & $-5,7760$ \\
\hline & $-10,6151$ & $-7,4775$ & $-11,1283$ & $-8,3360$ \\
\hline \multirow{2}{*}{ DLOG(EXP_FUEL) } & & $-1,1579$ & & \\
\hline & & $-1,7710$ & & \\
\hline \multirow{2}{*}{ DLOG(XC) } & & & & $-0,6113$ \\
\hline & & & & $-0,8822$ \\
\hline \multirow{2}{*}{ DLOG(XR) } & & & & 3.0845 \\
\hline & & & & 44515,8031 \\
\hline \multirow{2}{*}{ DLOG(OPEN) } & 7,2466 & & & \\
\hline & 10,6961 & & & \\
\hline \multirow{2}{*}{ DLOG(OPENC) } & & & $-0,6457$ & \\
\hline & & & $-0,9957$ & \\
\hline \multirow{2}{*}{ DLOG(OPENR) } & & & 5,9923 & \\
\hline & & & 9,2402 & \\
\hline
\end{tabular}

*Los valores resaltados corresponden al efecto a largo plazo.

En este trabajo se hicieron estimaciones que recogieran los efectos de la apertura económica y la afluencia de capitales. En el caso de la apertura se muestra que en algunos casos esta variable tiene un efecto positivo sobre el crecimiento y en otros casos su efecto no es significativo (Tabla 2).

En el caso de afluencia de capitales se usaron variables relacionadas como la inversión extranjera y la deuda externa, pero en ningún caso se pudo comprobar una relación estadísticamente significativa y por tanto no se pudo establecer si la afluencia de capitales generaba o no un efecto negativo sobre el sector transable y el crecimiento.

Adicionalmente se usaron otras medidas de exportaciones como proporción del PIB que incluyen a los combustibles (XC) y otra que los excluye (XR). Pero los efectos no son significativos. 


\section{CONCLUSIONES}

En las últimas décadas, las exportaciones de combustibles como el petróleo y carbón han generado efectos que modificaron la estructura del sector externo colombiano, han atraído importantes flujos de capital y han proporcionado ingresos fiscales muy significativos (Vargas, 2010).

El efecto sobre el crecimiento económico de las exportaciones de combustibles ha sido materia de debate en cuanto si ha sido una maldición o un beneficio para los países exportadores. La llamada enfermedad holandesa ha sido una hipótesis recurrente para explicar el efecto negativo de estas exportaciones sobre el desempeño negativo de la economía. Las bonanzas exportadoras han afectado el tipo de cambio, el sector transable de la economía y por tanto su crecimiento.

En Colombia se han hecho diferentes estimaciones sobre esta hipótesis y en algunos casos se encontró que las exportaciones de combustibles afectan el sector transable y el crecimiento, pero en otros casos el efecto no fue significativo. Sin embargo, algunos autores de la historia económica reciente del país aducen que las aperturas comercial y financiera afectaron el sector transable y los ciclos económicos.

Para analizar las hipótesis de la enfermedad holandesa, en este trabajo se estimó primero la relación entre las ex- portaciones de petróleo y carbón con la tasa de cambio, para establecer si estas exportaciones generaban la apreciación real que luego afecta la producción de transables, pero no se encontró una relación significativa. Por tanto, este canal de la enfermedad holandesa no se puede comprobar aquí.

Del mismo modo, se estimó la relación entre exportaciones combustibles con la producción de bienes transables y se encontró un efecto negativo, como predice la hipótesis de la enfermedad holandesa. En este mismo análisis se relacionó la producción de transables con medidas de apertura comercial y se encontró un efecto positivo. Asimismo, se intentó utilizar medidas de apertura financiera, pero no se pudo establecer una relación significativa de estas con la producción transable.

En cuanto a la relación entre exportaciones de combustibles y el crecimiento económico, se pudo comprobar un efecto negativo en algunas estimaciones, es decir, que el crecimiento de estas exportaciones afecta el crecimiento económico del país. Sin embargo, en otras ocasiones no se pudo comprobar esta relación. De modo que el efecto de estas exportaciones sobre el crecimiento sigue siendo materia de análisis.

\section{DECLARACION DE CONFLICTO DE INTERES}

Los autores declaran que no existe conflicto de intereses. 


\section{REFERENCIAS}

Clavijo, S., Vera, A. \& Fandiño, A. (2012). La desindustrialización en Colombia. Análisis Cuantitativo de sus determinanes. ANIF.

Corden, W. \& Neary, P. (1982). Booming Sector and De-Industrialisation in a Small Open Economy. Economic Journal, 92(386), 825-842. https://doi. org/10.2307/2232670

De Tray, D. (1994). Herramientas básicas para el análisis de una bonanza. Revista Planeación y Desarrollo, 25.

Ffrench-Davis, R. (1997). Flujos de capital y el desempeño de la inversión: una síntesis. En H. Reisen \& R. French-Davis, Flujos de capital e inversión productiva: Lecciones para América Latina (pp. 9-23). McGraw Hill.

Garay, L. (2013). Mineria en Colombia. Fundamentos para superar el modelo extractivista. Contraloría General de la Nación.

García, D. (2005). Economic Growth, Consumption and Oil Scarcity in Colombia: A Ramsey Model, Time Series and Panel Data Approach. Borradores del CIE, (12).

Gelb, A. (1988). Oil Windfalls. Blessing or Curse? http://documents.worldbank.org/ curated/en/536401468771314677/Oil-windfalls-Blessing-or-curse

Goda, T. \& Torres, A. (2015). Flujos de capital, recursos naturales y enfermedad holandesa: el caso colombiano. Ensayos sobre Política Económica, (33), 197206. https://doi.org/10.1016/j.espe.2015.07.001

Greco-Banco de la República. (2002). El crecimiento económico colombiano en el siglo $X X$. Fondo de Cultura Económica.

Haussman, R. \& Rigobon, R. (2003). An Alternative Interpretation of the 'Resource Curse': Theory and Policy Implications. https://doi.org/10.3386/w9424

Kalmanovitz, S. (2015). Nueva historia económica de Colombia. Taurus.

Montenegro, S., Suescún, R. \& Pardo, R. (2001). Petróleo, vulnerabilidad de la economía colombiana y políticas de estabilización. Universidad de los Andes.

Ocampo, J. (2015). Historia económica de Colombia. Fondo de Cultura Económica. 
Ojeda, J., Parra, J. \& Vargas, C. (2013). Flujos de capitales, choques externos y respuesta de políticas en países emergentes. En H. Rincón \& A. Velasco, Auge minero-energético en Colombia: efectos macroeconómicos y respuestas de política fiscal (pp. 565-567). Banco de la República.

Perla, C. (2005). ¿Cuál es el destino de los países abundantes en recursos minerales? Nueva evidencia sobre la relación entre recursos naturales, instituciones y crecimiento económico. Revista Economía, 27(53-54).

Perry, G. \& Olivera, M. (2012). Petróleo y minería: ¿Bendicion o maldición? La Imprenta Editores.

Piraján, J., Urrea, I. \& Gutiérrez, D. (2012). Enfermedad holandesa. El auge del sector minero-energético colombiano 2000-2010. https:/www.cesla.com/pdfs/ enfermedad $\% 20$ holandesa $\% 20$ CESLA.pdf

Posada, C. (1994). El petróleo de Cusiana, las perspectivas y la política económica. En A. Montenegro, Cusiana: un reto de politica económica (pp. 343-347). Departamento Nacional de Planeación.

Rodríguez, F. \& Sachs, J. (1999). Why do Resource Abundant Economies Grow More Slowly? A New Explanation and an Application to Venezuela. www. goodpracticemining.org/documents/jon2/Why.pdf.

Sachs, J. \& Andrew, W. (1997). Natural Resource Abundance and Economic Growth. Center for International Development and Harvard Institute for Internacional Development.

Urrutia, M. (1994). Economía politica del manejo de la bonanza petrolera. Revista Planeacion y Desarrollo, 25.

Vargas, J. (2010). Impacto de las exportaciones de hidrocarburos en el crecimiento económico colombiano 1970-2007. (Tesís de maestría). Universidad Nacional de Colombia. 